\title{
CORRESPONDENCE
}

\section{PAWN ODDS SHOOTOUTS}

\author{
Larry Kaufman
}

\section{Wooden Brıdge Road, Potomac, MD 20854 USA}

In order to evaluate David Levy's proposals for Pawn-Odds matches between computers and Grandmasters, (see ICGA Journal, Vol 28, No 2, p 111 ) I ran a series of matches using the "Shootout" feature of the PC programs I removed each of the white Pawns, one at a tıme, and ran ten-game matches at an "actıve" tıme limit (15'+10" increment) and at two blitz tıme limits (2'+1" and 3'+2"), using ten of the strongest PC programs on the market on a fast single-processor PC I then averaged the results at blitz and active chess, weightıng the actıve results double Here are the results

Results For the edge-Pawns, the results were nearly identical (35\% for "a" and $358 \%$ for "h") The combined result of $354 \%$ translates to 106 ratıng points For the "f"-Pawn, the score of $3333 \%$ translates to 120 ratıng points For all the remainıng five Pawns, the results were nearly the same (21 67\% for "c" and "d", $2333 \%$ for "b", "e", and "g") The combined result of $2267 \%$ translates to 213 rating points For all Pawns combined, the blitz results were $521 / 2-1071 / 2$ for a ratıng differential of 124 , while the active results were $191 / 2-601 / 2$ for a rating differential of 197 I also reran the blitz results with Black removing the Pawn instead of White, and the resultant score of 120-40 gives a rating differential of 191 So, it was 67 points more than when White removes the Pawn

Discussion The biggest surprise was that the "f"-Pawn turned out to be the smallest handicap of the non-edge Pawns, in marked contrast to the judgment of the programs themselves I suspect that this is because White usually castles to the kingside, and the Rook finds itself on a half-open file

Traditıonally, when Black removed a Pawn it was always the "f"-Pawn, thought to be the biggest handicap because Black encounters severe difficulties in developing normally and castling after 1 e4 However this does not apply when White gives the handicap The fact that the other non-edge Pawns had essentially the same results seems reasonable to me The Pawns increase in value towards the centre, but the removal of a centre Pawn helps development more than removal of any other Pawn, and the removal of a Knight's Pawn leaves the Rook's Pawn isolated These factors apparently cancel out

Conclusions Pawn handicap is a perfectly valid handicap, but there is no justification for any hierarchy other than the edge-Pawn and the non-edge Pawn My suggestion would be "a"-Pawn for the edge-Pawn and "c" for the non-edge Pawn, as these handicaps seem to distort the game less than the alternatives The "c"-Pawn handicap seems to be about double in magnitude to the "a"-Pawn handicap It should also be pointed out that the "a"-Pawn handicap is about equivalent to a twomove handicap White is worth about 35 points in Master play, so a tempo is worth 70 points (the difference between having White and having Black) and so White plus another tempo should be worth about 105 points, nicely matching the 106 points found in my study for the edge-Pawn Whether a two-move handicap or a White "a"-Pawn handicap makes a more interesting game is difficult to say I should also point out that instead of a hierarchy of Pawn handicaps, the study strongly supports the alternatıve proposal of using one Pawn and steadily increasing the time limit to increase the handicap

Magnitude The ratıng equivalence of the handicap clearly increases with longer tıme limits and with higher skill levels The increase from 124 to 197 points from blitz to active chess clearly proves this Extrapolating from these results, in round number I would expect that at 40/2 with contestants averaging 2750 (roughly the average of the programs tested) the "a" Pawn handicap should be around 150 points, and the "c"-Pawn around 300 With HYDRA performing at 3000 in ten games to date, it should be a slight favourite against any human at odds of the "a"-Pawn, and a farr match for a 2700-level GM at odds of the "c"-Pawn Against a 2600-level GM odds of the Black "c"-Pawn should be fair, and against a 2500-level GM the traditional "Pawn and Move", the Black "f"-Pawn, might be appropriate

A Coda: A player of GM strength played against three different top programs at a tıme control of all moves in 25 minutes, with an increment of 10 seconds per move In the first game, at traditional odds of Pawn and move, the GM won easily For the second game the program played White and gave the f2-Pawn - the game ended in a draw after the GM missed several clear wins in the endgame For the third game the program's c2-Pawn was removed, and this time the computer won' So it would seem that in rapid chess, Pawn odds (computer White) is indeed a reasonable handicap for a 2550 FIDE rated GM

Reference: The full story of this match, with the games annotated by the GM (Alex Wojtkiewicz) will appear in Chess Life magazıne soon 